\title{
Inhibidores del cotransportador sodio- glucosa tipo 2 (SGLT2). Una nueva generación de fármacos para control de diabetes mellitus tipo 2
}

\section{Artículo de revisión}

Rodríguez-Ramírez José Félix*, Robles-Álvarez Víctor Manuel*, Madrigal-Elizalde Emmanue**, García-Ayala Miguel Ángel*, Masuoka-lto David**, Terrones-Saldívar Ma del Carmen ***

\section{Resumen \\ - La diabetes es una de las enfermedades crónicas dege- \\ - nerativas con mayor prevalencia en el mundo. Los trata- \\ - mientos actuales no han sido suficientes para obtener el \\ - control metabólico de los pacientes. Recientemente se \\ - han desarrollado una serie de fármacos con mecanis- \\ - mo de acción diferente a los ya conocidos, denominados \\ - inhibidores del cotransportador de sodio y glucosa tipo \\ - 2 (SGLT 2) que han demostrado una serie de resulta- \\ - dos favorables, entre ellos el control de la glucosa sérica, \\ - disminución de peso y disminución de la presión arterial, \\ - entre otros. En México, la Comisión Federal para la Pro- \\ - tección contra Riesgos Sanitarios (COFEPRIS) aprobó la \\ - venta de estos fármacos para su aplicación terapéutica \\ - con la esperanza de que en el sector público comience \\ - a distribuirse a finales del 20 / 4. LUX MÉDICA, AÑ09, NÚMERO \\ - 27, MAYO-AGOSTO 2014, PP 23-30}

Palabras clave: SGLT2, diabetes mellitus, control glucé-

\section{Abstract}

Diabetes mellitus type 2 is one of the chronic degenerative diseases with the highest prevalence in the world. Current treatments have not been sufficient to obtain metabolic control of patients. A number of drugs with a mechanism of action different from the already known, has recently been developed called the co-transporter of sodium and glucose inhibitors type 2 (SGLT-2) that have shown a series of favorable results, among them control serum glucose, weight reduction and decrease of blood pressure, among others. In Mexico, the Federal Commission for the Protection Against Sanitary Risks, approved the sale of these drugs for therapeutic application in the hope that in the public sector begins to be distributed by the end of 20 / 4. LUX MÉDICA, AÑO 9, NÚMERO 27, MAYO-AGOSTO 2014, PP 23-30

Key words: SGLT2, diabetes mellitus type 2, glycemic control

- Estudiantes del noveno semestre de la carrera de Medicina del Centro de Ciencias de la Salud de la Universidad Autónoma de Aguascalientes.

** Profesor investigador del Departamento de Estomatología del Centro de Ciencias de la Salud de la Universidad Autónoma de Aguascalientes

*** Profesora investigadora del Departamento de Gineco-Obstetricia y Pediatría del Centro de Ciencias de la Salud de la Universidad Autónoma de Aguascalientes.

Fecha de recibido: 20 junio 2014

Fecha de aceptación: 21 de julio 2014

Correspondencia: David Masuoka Ito DDS, MMM, PhD. Departamento de Estomatología. Centro de Ciencias de la Salud. Universidad Autónoma de Aguascalientes. Avenida Aguascalientes No 940, Unidad Médico Didáctica, Edificio 101, Ciudad Universitaria. Aguascalientes, Ags., México CP 20131 Teléfono +52(449)9108438. Correo electrónico david.masuoka@gmail.com 


\section{Introducción}

\section{Generalidades}

La diabetes mellitus es un problema de salud pública debido a su creciente prevalencia, las complicaciones que presentan los pacientes que la padecen y porque representa una de las principales causas de muerte en nuestro país.

Una estimación de la OMS indica que a nivel mundial, desde 1985 a 2013 casi se ha triplicado el número de casos de diabetes, con cifra actual estimada en más de 366 millones de personas con diabetes. ${ }^{1-4}$ La Federación Internacional de Diabetes (FID) estima que para el 2030 habrá 552 millones de casos de diabetes. ${ }^{5}$ En México, según la Encuesta Nacional de Salud y Nutrición (ENSANUT) 2012, la prevalencia de diabetes mellitus en nuestro país es de $9.2 \%$ y sólo el $25 \%$ de los adultos diabéticos están en control metabólico, ocupando el sexto lugar mundial en pacientes con diabetes según la FID..$^{5-7}$ Se calcula que los 6.8 millones de pacientes con diagnóstico de diabetes mellitus en el 2013 aumentará a 11.9 millones en el $2030 .^{7}$

Todas las sociedades científicas consideran adecuado el tratamiento escalonado, en el que se encuentra recomendado como primer paso, realizar cambios en el estilo de vida (alimentación saludable, ejercicio físico, educación nutricional, abstención del tabaquismo). ${ }^{8}$ En la actualidad hay una gran diversidad farmacológica para el tratamiento de los pacientes diabéticos (ver tabla 1), que se prescriben de acuerdo a indicaciones precisas, sin embargo, en no pocas ocasiones, con el tratamiento hipoglucemiante no se alcanza el control metabólico, además de que algunos de ellos tienen como efectos colaterales el riesgo de hipoglicemia e incremento de peso corporal, manteniendo la fisiopatología de la resistencia a la insulina. ${ }^{4}$

\section{Tabla I}

Principales fármacos utlizados para el tratamiento de la diabetes mellitus tipo 2. 4,8

\begin{tabular}{|c|c|c|c|}
\hline \multicolumn{2}{|r|}{ Clase } & Ejemplo & $\frac{\text { Mecanismo de acción }}{\text { Activa la AMP-cinasa, aumenta la sensibilidad a la insulina. }}$ \\
\hline 2 & Sulfonilureas & Glibenclamida & $\begin{array}{l}\text { Cierra los canales de KATP de las células } \beta \text {, aumenta la secreción de } \\
\text { insulina }\end{array}$ \\
\hline 3 & Metiglinidas & Repaglinida & $\begin{array}{l}\text { Cierra los canales de KATP de las células } \beta \text {, aumenta la secreción de } \\
\text { insulina }\end{array}$ \\
\hline 5 & $\begin{array}{l}\text { Inhibidores de } \\
\alpha \text {-glucosidasa }\end{array}$ & Acarbosa & Inhibe la $\alpha$-glucosidasa intestinal, aumenta la sensibilidad a la insulina \\
\hline 6 & $\begin{array}{l}\text { Agonistas del receptor } \\
\text { GLP-1 }\end{array}$ & Exenatida & $\begin{array}{l}\text { Activa los receptores GLP-1 de las células } \beta \text {, aumenta la } \\
\text { secreción de insulina }\end{array}$ \\
\hline 7 & Inhibidores de DPP-4 & Sitagliptina & $\begin{array}{l}\text { Inhibe la actividad de DDP-4, aumenta la sobrevida de } \\
\text { incretinas }\end{array}$ \\
\hline
\end{tabular}


Todos los fármacos utilizados en el tratamiento de la diabetes mellitus tienen como primer objetivo disminuir las cifras de glucosa en sangre, sin embargo, esto no se logra en todos los casos. Como ejemplo de lo anterior se encuentra publicado el resultado de una encuesta realizada en Estados Unidos entre 2003-2006, que reportó que sólo el $57.1 \%$ de los pacientes diabéticos en tratamiento, lograron un control óptimo de la glucosa. ${ }^{9}$ En fechas recientes las cifras son menos alentadoras, Yu-nan (2014) encontró que aproximadamente dos tercios de los pacientes diabéticos de Europa y Estados Unidos tratados con esquemas tradicionales, no logran un control glucémico. ${ }^{10}$

\section{Homeostasis de la glucosa por el riñón}

El riñón realiza una importante función en la homeostasis de la glucosa, a través de dos mecanismos: el primero corresponde a la gluconeogénesis (contribuye con el $20 \%$ de la producción total de glucosa) y el segundo a la filtración glomerular y reabsorción de la glucosa en los túbulos contorneados proximales del riñón. ${ }^{11-13}$

Los glomérulos de un adulto sano filtran y reabsorben aproximadamente $180 \mathrm{gr}$ de glucosa cada día. En circunstancias normales casi toda esta glucosa se reabsorbe y menos $1 \%$ se excreta en la orina. ${ }^{4,12,14} \mathrm{El}$ $90 \%$ de la glucosa filtrada es reabsorbida por el co-transportador de sodio-glucosa tipo 2 (SGLT2) en el segmento contorneado del túbulo proximal, el 10\% restante se reabsorbe por el co-transportador de sodio-glucosa tipo 1 (SGLT1) en el segmento recto del túbulo proximal descendente. Cuando la carga de glucosa tubular es de aproximadamente $120 \mathrm{mg} / \mathrm{min}$ o menos, no se pierde la glucosa en la orina. Sin embargo cuando la carga de glucosa excede aproximadamente $180-200 \mathrm{mg} / \mathrm{dl}$ (el denominado "umbral de la glucosa"), la glucosa comienza a aparecer en la orina. ${ }^{12}$

En la absorción de la glucosa a nivel renal participan una familia de proteínas transmembrana denominadas cotransportadores de sodio-glucosa, de las cuales se conocen siete isoformas. La caracterización de los cotransportadores SGLT1 y SGLT2 se muestran en tabla 2.12,13 Tanto SGLT1 y SGLT2 se encargan de la reabsorción de la glucosa del filtrado glomerular y se encuentran localizados en el túbulo contorneado proximal de la nefrona, sin embargo es SGLT2 el que principalmente se encarga de esta función (figura1). La reabsorción de glucosa a través de la membrana celular se realiza mediante un transporte activo, que utiliza al sodio como sustrato, y a la bomba de $\mathrm{Na}+/ \mathrm{K}+$ ATP asa. ${ }^{15}$
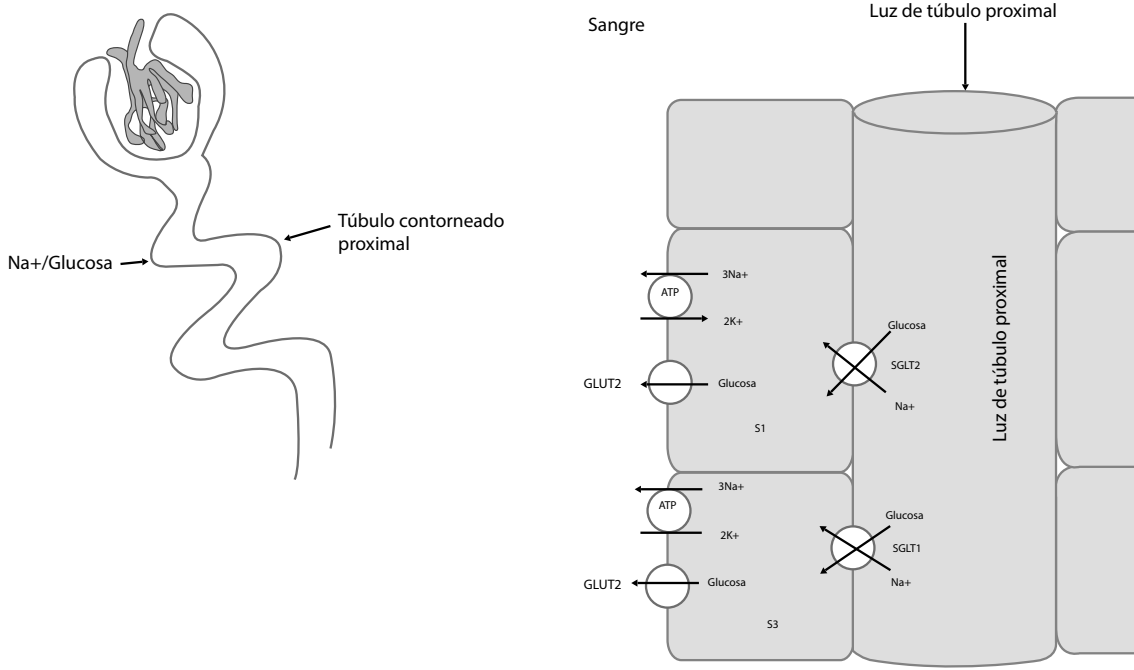

Fig. 1 Modelo del transportador $\mathrm{Na+l}$ Glucosa en riñón. El transportador SGLT2 se expresa predominantemente en el segmento S1 del túbulo contorneado proximal y es responsable de la mayoría de la reabsorción renal de glucosa. $^{13}$ 


\section{Tabla 2}

\section{Comparación de los transportadores SGLT1 y SGLT2 12}

\begin{tabular}{|lll|}
\hline \multicolumn{1}{|c|}{ SGLT1 } & SGLT2 & \\
\hline Localización & $\begin{array}{l}\text { Intestino delgado, en menor cantidad } \\
\text { en riñón, cerebro y corazón }\end{array}$ & $\begin{array}{l}\text { Riñón, en menor cantidad en cerebro, hígado, } \\
\text { tiroides, músculo esquelético, corazón }\end{array}$ \\
\hline Sustancia transportada & Glucosa o galactosa & Glucosa \\
\hline Afinidad por la glucosa & Alta & Baja \\
\hline $\begin{array}{l}\text { Capacidad de transporte } \\
\text { de glucosa }\end{array}$ & Baja & Alta \\
\hline $\begin{array}{l}\text { Reabsorción tubular } \\
\text { de glucosa (\%) }\end{array}$ & $10 \%$ & $90 \%$ \\
\hline Función & $\begin{array}{l}\text {-Absorción intestinal de glucosa } \\
\text { y galactosa } \\
\text {-Reabsorción renal de glucosa }\end{array}$ & $\begin{array}{l}\text {-Reabsorción renal de glucosa } \\
\text { en el segmento S1 del túbulo proximal }\end{array}$ \\
\hline
\end{tabular}

En los pacientes con DM2 existe un incremento de la expresión y actividad de SGLT2 ${ }^{13}$, dando como resultado aumento en el transporte máximo de glucosa entre 20 a $40 \%$ en personas con diabetes tipo 2 en comparación con personas sanas. ${ }^{9,15}$

Recientemente se ha agregado una nueva generación de fármacos que ayuda a mejorar los niveles de glucosa sérica, y los niveles de $\mathrm{HbA} 1 \mathrm{C}$, ofreciendo a los diabéticos la posibilidad de un excelente control de su padecimiento, son los inhibidores de co-transportador sodio-glucosa tipo 2 (SGLT2). ${ }^{16-20}$

Para lograr sintetizar estos fármacos se partió de dos observaciones: la primera corresponde a que ya se sabía que los pacientes con glucosuria renal familiar presentan una mutación en el gen del cotransportador SGLT2, lo que les ocasiona una eliminación diaria de más de 100 gr de glucosa por orina, sin presentar repercusiones mayores en los pacientes, ya que el sodio del filtrado glomerular se reabsorbe por otros transportadores. La segunda observación se relaciona con un compuesto natural aislado de la corteza del manzano desde 1835, la florizina, utilizada inicialmente en el tratamiento de la fiebre y las enfermedades infecciosas. Medio siglo después observaron que altas dosis de florizina causaban glucosuria. En 1970 se descubrió que la propiedad glucosúrica de la florizina se debe a un efecto inhibidor del transportador de sodio-glucosa tipos 1 y $2 .{ }^{4}$ Diez años más tarde, se descubrió que la florizina normalizaba la glucemia en ayuno y pospandrial en el $90 \%$ de los animales pancreatectomizados por inducción de la glucosuria. Durante la década de los noventa se realizó la clonación de los dos transportadores. Sin embargo, el uso de la florizina como agente hipoglucemiante se vio limitado debido a que su inhibición no es selectiva, inhibe tanto a SGLT2 como a SGLT1, por lo que ocasiona como efecto colateral diarrea osmótica. 3,4,9 Este conocimiento desprende el interés por sintetizar análogos de la florizina pero selectivos de los SGLT2.

\section{Inhibidores del transportador SGLT2 para el tratamiento de la diabetes mellitus tipo 2}

En la actualidad se han desarrollado fármacos análogos de florizina selectivos para SGLT2, de los cuales la dapagliflozina y la canagliflozina son los más desarrollados. ${ }^{12}$ Existen diferentes fármacos de esta familia, algunos ya aprobados por diferentes agencias internacionales (ver tabla 3 ). Los inhibidores del cotransportador de sodio- 
glucosa tipo 2, son un grupo reciente de hipoglucemiantes, que inhiben el SGLT2 en el túbulo proximal renal, aumenta la excreción renal de glucosa y secundariamente disminuye los valores plasmáticos de ésta, con bajo riesgo de hipoglicemia. En humanos, los inhibidores del SGLT2 in- hiben entre el 30 y $50 \%$ de la reabsorción renal de glucosa.9,20,21 Otro beneficio que se ha reportado por el uso de estos fármacos es que debido a la pérdida de glucosa por la orina, que representan calorías, pueden inducir pérdida de peso. Es importante resaltar que el mecanismo de acción

\section{Tabla 3}

\section{Fases de desarrollo de los diferentes inhibidores de SGLT2 ${ }^{9,12,22-24}$}

\begin{tabular}{|ll|}
\hline Fármaco & Fase de desarrollo \\
\hline Dapagliflozin & Aprobado por la Agencia Europea de Medicina, la FDA y en México \\
\hline Canagliflozin & Aprobado por la FDA. \\
\hline Empagliflozin & Aprobada por la Agencia Europea de Medicina. \\
\hline Ipragliflozin & Aprobado en Japón. \\
\hline Luseogliflozin & Aprobada en Japón. \\
\hline Tofogliflozin & Fase 3 \\
\hline Ertugliflozin & Fase 2 \\
\hline LX 4211 & Fase 2 \\
\hline EGT0001442 & Fase 2 \\
\hline GW 869682 & Fase 2 \\
\hline ISIS 388626 & Fase 1 \\
\hline
\end{tabular}

de estos fármacos es independiente de la función de las células beta del páncreas. ${ }^{20}$

La Agencia de Medicina Europea ha recomendado a la dapagliflozina, un inhibidor SGLT2, para el tratamiento de la diabetes tipo 2. La dapagliflozina ha mostrado en múltiples ensayos clínicos aleatorizados, que reduce las concentraciones plasmáticas de glucosa en ayuno y postprandial, la HbA1cy el peso corporal, con bajo riesgo de hipoglicemia. Además es bien tolerada, $y$ tiene como efecto colateral poliuria y sed, aunque se ha descrito un aumento de las infecciones del tracto genital y urinario. ${ }^{8}$

\section{Dapagliflozina}

La dapagliflozina es el primer fármaco que pertenece a un grupo catalogado como inhibidores de SGLT2 que han sido propuestos y en algunos países ya aprobados para el tratamiento de pacientes con diabetes mellitus tipo 2. La dapagliflozina es una sustancia química que inhibe a SGLT2 de forma selectiva y reversible, ocasionando la eliminación de aproximadamente 70 gr de glucosa por orina al día.

Tiene una vida media de 8.1 a 12.2 horas. La dapagliflozina, después de su administración oral, se absorbe en un tiempo promedio de 1 hora, se metaboliza mediante reacciones de fase II, a través de la conjugación con la glucuronil transferasa, que produce un metabolito inactivo. Su excreción renal es baja. ${ }^{12}$

Recientemente, un grupo de investigadores chinos, interesados en conocer las evidencias sobre la seguridad y eficacia de la dapagliflozina realizaron y publicaron una revisión sistemática ${ }^{25}$ que incluyó diez estudios clínicos aleatorizados (ECA), realizados entre 2009 y 2012, de ocho países. Para seleccionar dichos ECA, buscaron estudios primarios sobre dapagliflo- 
zina utilizada como monoterapia en dosis que variaron entre 1 y $50 \mathrm{mg}$ por día, o en adición con otros hipoglicemiantes, en hombres y mujeres con diabetes mellitus tipo 2, mayores de 18 años, tratados por lo menos durante 12 semanas (grupo tratado con dapagliflozina $n=3464$ pacientes, grupo placebo $n=1331$ ). Cuantificaron como medida de eficacia la $\mathrm{HbA} 1$, la glicemia y la pérdida de peso. Para evaluar la seguridad evaluaron la frecuencia de hipoglicemia y la frecuencia de infecciones del tracto génito-urinarias. Los resultados en todos los estudios mostraron una disminución de la $\mathrm{HbA} 1 \mathrm{c}$ en el grupo tratado con dapagliflozina, en un rango entre 0.39 y $2.05 \%$, disminución significativa comparada con el grupo placebo. Los resultados no fueron diferentes cuando compararon los niveles de $\mathrm{HbA} 1 \mathrm{c}$ en los pacientes tratados con dapagliflozina versus metformina. Respecto al control de la glicemia se observó una disminución significativa en el grupo tratado comparado con el grupo placebo. En el grupo tratado, encontraron que los pacientes mostraron una disminución del peso corporal entre 0.69 y $8.54 k$, esta disminución fue significativa cuando se comparó con el grupo placebo así como contra el grupo de metformina. No encontraron hipoglicemia en el grupo tratado con dapagliflozina como monoterapia, pero sí se encontró el incremento en el riesgo de infecciones urinarias $(R R=1.33)$ y genitales $(R R=3.23)$. La presión arterial mostró una disminución tanto de la sistólica como de la diastólica en el grupo tratado. Se reportaron como efectos colaterales tos y cefalea.

En otra revisión sistemática publicada también en este año ${ }^{10}$, que incluyó doce ensayos clínicos aleatorizados, con un total de 3986 pacientes, compararon grupos de pacientes tratados con dapagliflozina combinada a la metformina versus placebo más metformina, o dapagliflozina más insulina versus placebo más insulina, con un seguimiento entre 12 a 104 semanas. Encontraron que la adición de la dapagliflozina al tratamiento convencional de la diabetes mejoró el control en los niveles de la hemoglobina glucosilada (HbA1c) y de los niveles glicémicos en comparación con el grupo control, además encontraron que el grupo de pacientes diabéticos tratados con dapagliflozina redujeron peso corporal, condición favorable para el control metabólico de estos pacientes.

Podemos resumir que los inhibidores del SGLT-2 se asocian con una reducción de la $\mathrm{HbA} 1 \mathrm{c}$ en un $0.6-0.9 \%,{ }^{12,13,26}$ se asocian con una pérdida calórica diaria de 100-300 Kcal lo cual ayuda a la baja de peso $^{12,21,27,28}$ y la presencia de una leve diuresis osmótica puede contribuir al control de la presión (reducción presión sistólica de 2-10 $\mathrm{mmHg}$ ), disminuyendo un factor de riesgo cardiovascular..$^{9,12,21}$ Se observan buenos resultados en pacientes con cualquier grado de resistencia a la insulina o disfunción de células $\beta$ pancreáticas ${ }^{12,13,21}$ y otro de sus beneficios es que no desarroIlan hipoglucemias. 9,13,21,27,29-32

La dapagliflozina ha sido aprobada en algunos países como México, Estados Unidos y la unión Europea mientras que la luseogliflozina ha sido aprobada para su uso en Japón. ${ }^{33-36}$ La canagliflozina es otro inhibidor SGLT2, aprobado por la Food and Drug Administratio (FDA, por sus siglas en inglés) así como por la Comisión Federal para la Protección contra Riesgos Sanitarios (COFEPRIS) en nuestro país, como un fármaco para el control y tratamiento de la diabetes mellitus tipo 2 en adultos.

La revisión de la evidencia disponible, nos muestra que el inhibidor de SGLT2 de mayor ventaja respecto a los demás de su grupo es la dapagliflozina, que se perfila como otra alternativa en el tratamiento de los pacientes con diabetes mellitus tipo 2 . 


\section{Conclusiones}

Los resultados de las revisiones sistemáticas indican que la dapagliflozina tiene beneficios en el control metabólico en los pacientes diabéticos tipo 2. Los efectos adversos encontrados varían de leves a moderados; sin embargo, es necesario realizar ensayos clínicos aleatorios que tengan un seguimiento a largo plazo para identificar la seguridad de los inhibidores de SGLT2 y establecer la verdadera utilidad de estos fármacos en el tratamiento de los pacientes diabéticos.

\section{Bibliografía}

1. International Diabetes Federation: IDF Diabetes Atlas. 5 th ed. Brussels, Belgium, International Diabetes Federation, 2011. Available from http://www. idforg/diabetesatlas.

2. Hernández M, Gutiérrez JP, Reynoso N. Diabetes mellitus en México. El estado de la epidemia. Salud Pública Méx 2013;55 supl 2: S129-S136.

3. Anandi P. El transportador SGLT2. (Sodium GLucosa co-Transporter-2. Revista Médica Uruguay, opción medica 35, 2013

4. Galindo-Capos M, Carrillo-Ocampo L, Cortazár-Benítez F, Aisa-Álvarez A, et al. Inhibidores del transportador de sodio-glucosa tipo 2 (SGLT2) en el tratamiento de pacientes con diabetes mellitus: el control glucémico a traves de la glucosuria. Med Int Mex. 2013; 29: i-v.

5. Han-Cho N, Whiting D, Guariguata L, AschnerMontoya P, Forouhi N, et al. IDF diabetes atlas. International diabetes Federation. 6 ed. 2013. P. 1417.

6. Encuesta Nacional de salud y nutrición 2012. Consultada en ensanut.insp.mx/informes/ENSANUT2012ResultadosNacionales.pdf.

7. Gil LE, Sil MJ, Aguilar L, Et al. Perspectiva de la diabetes mellitus tipo 2 en el instituto mexicano del seguro social. Rev Med Inst Mex Seguro Soc. 2013;51: 5867.

8. Ascaso JF. Diabetes mellitus tipo 2: nuevos tratamientos. Med Clin (Barc). 2013.http://dx.doi. org/10.1016/j.medcli.2013.05.041.

9. Chao EC. SGLT-2 Inhibitors: A New Mechanism for Glycemic Control. Clinical Diabetes. January 2014; 32: 4-11.

10. Yu-nan Sun, Yi Zhou, Xi Chen, Weng-si Che, Siuwai Leung. Evidence based practice - Research: The efficacy of dapagliflozin combined with hypoglycaemic drugs in treating type 2 diabetes mellitus: meta-analysis of randomised controlled trials BMJ Open 2014;4:e004619. doi:10.1136/bmjopen-2013-004619.
11. White JR. Apple Trees to Sodium Glucose Co-Transporter Inhibitors: A Review of SGLT2 Inhibition. CLINICAL DIABETES. 2010; 28(1):5-10.

12. Pérez-López G, González-Albarrán $O$, Cano-Megías M. Inhibidores del cotransportador sodio.glucosa tipo 2 (SGLT2): de la glucosuria renal familiar al tratamiento de la diabetes mellitus tipo 2. Revista de nefrología 2010;30(6):618-625.

13. Valentine V. The Role of the Kidney and SodiumGlucose Cotransporter-2 Inhibition in Diabetes Management. Clinical Diabetes. 2012; 30(4):151-155.

14. Nair S, Wilding P. Sodium glucose cotransporter 2 inhibitors as a new treatment for diabetes mellitus. J.Clin Endocrinol Metab 2010;95:34-42.

15. Clar C, Gill JA, Court R, Waugh N. Systematic review of SGLT2 receptor inhibitors in dual or triple therapy in type 2 diabetes. BMJ Open.2012;2:e001007. doi:10.1136/bmjopen-2012-001007.

16. Anderson S, Marrs J. Dapaglifozin for the treatment of type 2 diabetes. Ann Pharmacother. 2012; 46: 590-598.

17. Bailey CJ, Day C. SGLT2 inhibitors: glucoretic treatment for type 2 diabetes. British Journal of Diabetes \& Vascular Disease. 2010; 10: 193-199.

18. Monami M, Nardini C, Mannucci E. Efficacy and safety of sodium glucose co-transport-2 inhibitors in type 2 diabetes: a meta-analysis of randomized clinical trials. Diabetes, Obesity and Metabolism. September 2013; 16: 457-466.

19. Zhang L, Feng $Y$, List J, Kasichayanula S, Pfister $M$. Dapagliflozin treatment in patients with different stages of type 2 diabetes mellitus: effects on glycaemic control and body weight. Diabetes, obesity and metabolism, Volume 12, Issue 6, June 2010; p. 510516.

20. Salsali A, Hruba $V$, mansfield $t$, Rohwedder $K$, Wessman C, et al. Efficacy Increases with Increasing Baseline HbA1c Category with Dapagliflozin Therapy. September 2013; p. 14-11.

21. Liu J, TaeWeon L, De Fronzo RA. Why do SGLT2 inhibitors inhibit only $30-50 \%$ of renal glucose reabsorption in humans?. DIABETES. 2012; 61: 21992204. 
22. Vercruysse $F$, Charpentier $G$, Wilding JP, Hollander P, González-Gálvez G. Eficacia y Seguridad de Canagliflozina en Sujetos con Diabetes Mellitus Tipo 2 Inadecuadamente Controlados con Metformina Más Sulfonilurea durante 52 Semanas. Asociación Europea para el estudio de la diabetes. Septiembre 2013;15(4):372-382.

23. Uehara A. Filling for marketing approval of SGLT2 inhibitor lusegliflozin hydrate (TS-071) in Japan. Taisho Pharmaceutical Holdings Co.; Ltd. April 2013, 3-24-1.

24. AstraZéneca, Bristol-Myers Squibb. Efficacy and safety of dapagliflozin, added to therapy of patients with type 2 diabetes with inadequate glycemic control on insulin (artículo online) http://clinicaltrials. gov/show/NCT00673231.

25. Mei Zhang, Lin Zhang, Bin Wu, Haolan Song, Zhenmei An, Shuanging Li. Dapagliflozin treatment for 2 diabetes: a systematic review and meta-analysis of randomized controlled trials. Diabetes Metab Res Rev 2014;30:204-221 doi:10.1002/dmrr.2479.

26. Rosenstock J, Marisa V, Li W, Afshin Salsali, James F. List. Effect of Dapagliflozin, an SGLT2 inhibitor, on $\mathrm{HbA} 1 \mathrm{C}$, body weight, and hypoglucemia risk in patients with Type 2 Diabetes inadequately controlled on Pioglitazone monotherapy. Diabetes Care. 2012; 35: $1473-1478$.

27. Santer R, Calado J. Familial Renal Glucosuria and SGLT2: From a Mendelian Trait to a Therapeutic Target. Clin J Am Soc Nephrol. 2010; 5: 133-141.

28. Ovando $M$, Day $C$, inhibitors SGLT2: glucuretic treatment for type 2 diabetes. diabetes and vascular disease 2012 10: 193.

29. European Medicines Agency. Forxiga, dapagliflozin. Science Medicines Health. November 2012; (310) No. 1-3.
30. Bailey CJ, Gross JL, Pieters A, Bastien A, List JF. Effect of dapagliflozin in patients with type 2 diabetes who have inadequate glycaemic control with metformin: a randomised, double-blind, placebo-controlled trial. The Lancet, Volume 375, Issue 9733, June 2010; p. $2223-2233$

31. Shah NK1, Deeb WE, Choksi R, Epstein BJ. Dapagliflozin: a novel sodium-glucose cotransporter type 2 inhibitor for the treatment of type 2 diabetes mellitus. January 2012;32(1):80-94.

32. Brooks AM, Thacker SM. Dapagliflozin for the treatment of type 2 diabetes. Epub July 2009; 43(7):1286-93

33. Seino $Y$, Sasaki T, Fukatsu A, Samukawa $Y$, Sakai S Luseogliflozin (TS-071), a selective SGLT2 inhibitor, improves glycaemic control and lowers body weight in a 12-week study in Japanese patients with type 2 diabetes mellitus. Oasis. October 2012; p. 5-6.

34. Takada T. Filing for Marketing Approval of SGLT2 Inhibitor Luseogliflozin Hydrate (TS-071) in Japan. Taisho Pharmaceutical Holdings. April 2013; p. 1-2.

35. Seino Y, Sasaki T, Fukatsu A, Sakai S. et al. Efficacy and safety of luseogliflozin monotherapy in Japanese patients with type 2 diabetes mellitus: a 12-week, randomized, placebo-controlled, Phase II study. Current Medical Research \& Opinion. March 2014; $1-12$.

36. Seino Y, Sasaki T, Fukatsu A, Sakai S. et al. Dose-finding study of luseogliflozin in Japanese patients with type 2 diabetes mellitus: a 12-week, randomized, double-blind, placebo-controlled, phase II study. Current Medical Research \& Opinion. April 2014; p. $1-12$. 